\title{
Advances in inflammatory bowel disease: Are patients taking their medicines?
}

\author{
Subrata Ghosh MD FRCP FRCPE1 ${ }^{1}$, Paul C Adams MD FRCPC² ${ }^{2}$ Editor-in-Chief
}

$D^{\prime}$ Subrata Ghosh is the Professor of Gastroenterology at the Imperial College, Hammersmith Hospital, London, England. He has bench-to-bedside research interests in inflammatory bowel disease (IBD) and other intestinal inflammatory diseases. He will become the Head of the Division of Gastroenterology at the University of Calgary, Calgary, Alberta, in March 2009.

PA: There have been many advances in the therapeutics of IBD. The complexity of treatment has been vexing for many gastroenterologists. How are patients keeping up with the changing scene?

SG: Improved understanding of the immunopathogenesis of IBD has resulted in an explosion of translational research and clinical trials. Not only are there more therapeutic options, especially monoclonal antibody therapies, but there has also been a shift in the paradigm of treatment from short-term symptomatic treatment to

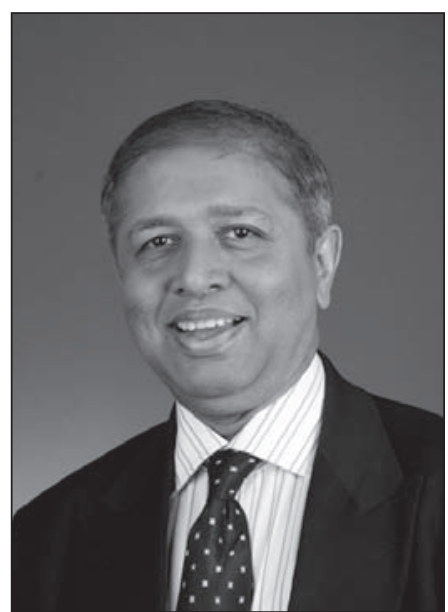

Dr Subrata Ghosh will become the Head of the Division of Gastroenterology at the University of Calgary, Calgary, Alberta, in March 2009

nonadherence rates ranging from $43 \%$ to $72 \%$ of IBD patients. In the United Kingdom, approximately $15 \%$ of patients fail to even redeem prescriptions at the pharmacy.

Treatment nonadherence rates vary considerably among countries. Within Europe, a survey (2) of 203 IBD patients revealed selfreported nonadherence rates ranging from $13 \%$ in France to $26 \%$ in Italy, 33\% in the United Kingdom and $46 \%$ in Germany. The overall nonadherence rate across Europe was 29\%.

PA: Is there a difference in patient adherence in symptomatic patients compared with maintenance therapy patients?

SG: Patients who are symptomatic and patients who have more severe disease adhere to their medications better. Unmarried patients, patients in full-time employment, depressed patients and patients who are prescribed medications three times daily do not adhere to their regimen as well. Adherence long-term disease modification. For patients, increasing options are always welcome. However, treatment of complex IBD patients has become a subspecialty, and patients are managed by a multidisciplinary team consisting not only of gastroenterologists, but also surgeons, pathologists, specialist nurses, pharmacists and dietitians. This is necessary to keep patients, and sometimes parents of affected children, well informed about treatment options, their benefits and especially potential adverse events. Patient organizations, as well as the industry, play an important role in patient education. However, our recent large European survey suggest that patients feel that doctors do not often discuss the impact of disease on their quality of life (1). It is important, therefore, to continue to uphold the patients' quality of life as central to patient management. Such a physician attitude will ensure that patients are given informed choice. Expert patients may also help.

PA: We know about nonadherence with medications through pioneering studies in hypertension and other diseases. What do we know about nonadherence in IBD patients?

SG: Within the controlled environment of clinical trials, in which there is good medical supervision, approximately $70 \%$ to $95 \%$ of patients with IBD adhere to their medication. However, this high rate of medication adherence is not reflected in dayto-day clinical practice. A cross-sectional study of outpatients in the United States (2) with quiescent ulcerative colitis found that only $40 \%$ were adherent to maintenance mesalazine therapy. Several community-based studies have reported starts to tail off approximately three months after commencement of therapy. It is unclear whether the current emphasis on chemoprevention of colorectal neoplasia by 5 -aminosalicylic acid compounds may improve long-term adherence.

PA: In Canada, many patients still have to pay for some of these medications. How does the rising costs of medications affect patient adherence and should this be a factor for the physician when choosing drug therapies?

SG: Costs borne by patients are always a factor in adherence. High-cost drugs, if borne by the patient, will lead to lower adherence. It is important that physicians keep in mind the possibility that a modest improvement in efficacy offered by a higher cost drug may be completely offset by poorer adherence. Such problems are often brought out by a sympathetic consultation.

PA: If you read the advertising, we may be offering medications that are more targeted, are taken less frequently, are more effective or have fewer side effects.

SG: In general, these are relevant advantages. There is good evidence that three times daily dosing of 5-aminosalicylic acid compounds leads to poorer adherence than once or twice daily. Fewer tablets may also offer advantages. However, most, if not all 5-aminosalicylic acid compounds may be administered once daily and current practice will increasingly offer once-daily dosing.

PA: How do we monitor nonadherence in an asymptomatic patient?

SG: Self-reporting only identifies $66 \%$ of patients who are

${ }^{1}$ Department of Gastroenterology, Imperial College, Hammersmith Hospital, London, England; ${ }^{2}$ University Hospital, London, Ontario

Correspondence: Dr Subrata Ghosh, Imperial College, Hammersmith Hospital, London, England W12 0NN.

Telephone 44-208-383-3266, fax 44-208-749-3436, e-mail s.ghosh@imperial.ac.uk 


\author{
TABLE 1 \\ Improving patient adherence to medication in \\ inflammatory bowel disease (IBD)

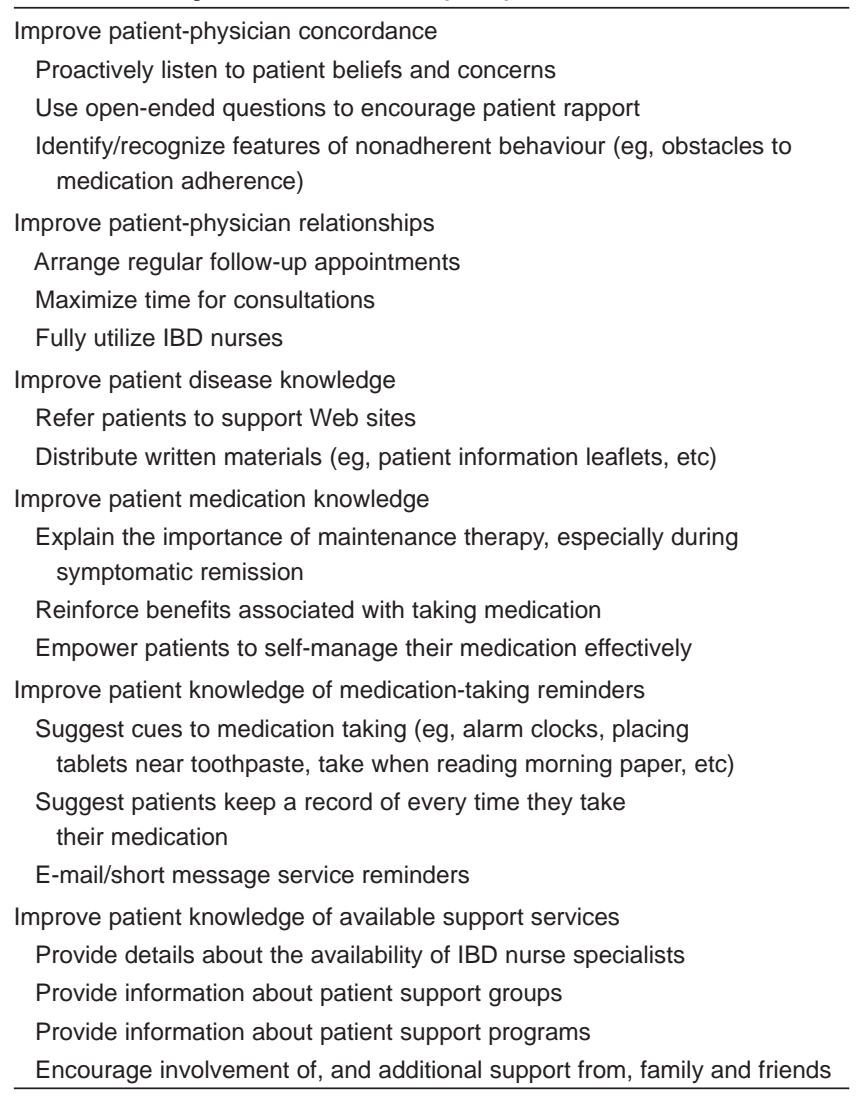

Data from reference 4

nonadherent to their 5-aminosalicylate medications as demonstrated by urinary excretion of the drug. Although urinary salicylate levels correlate with urinary 5-aminosalicylic acid levels, and is an inexpensive assay, it is not commonly used in clinical practice. In clinical practice, identifying patient profiles that are likely to be associated with a higher risk of nonadherence may alert physicians to explore adherence on a regular basis. In case of clinical relapses, nonadherence as an underlying cause always needs to be established. In patients on azathioprine or 6-mercaptopurine, measurement of metabolites, and monitoring mean corpuscular volume and leukocyte counts may also offer clues to poor adherence.

PA: With biological therapy, we may have the drug administered to the patient in an infusion and a record of the patient's compliance.

SG: There are patients who are at a risk of nonadherence and such patients undoubtedly may be suitable for intravenous administration of a biological therapy under supervision. On the other hand, some patients prefer the flexibility offered by home administration of a subcutaneous injection that saves them time and travel. Patient preference and patient profiling should guide choice of therapy. Most patients with IBD are young and would like to remain in employment where the flexibility of subcutaneous administration may be preferred. It is important to discuss the options with patients and offer them the choice. Even in patients on self-administered subcutaneous injections, regular follow-up every two to four months is necessary. Gaps in therapy may result in increased immunogenicity and loss of response.

PA: The asymptomatic patient often asks the physician "What is the end point for stopping the medications?"

SG: Clinical remission is no longer the end point and the focus has shifted to long-term disease modification (3). Such end points have not been robustly established. In ulcerative colitis patients, all are recommended to remain on 5-aminosalicylic acid to prevent relapses and development of neoplasia. In IBD patients on immunomodulator drugs (azathioprine, 6-mercaptopurine, methotrexate), my personal practice is to offer discontinuation after two to four years of relapse-free remission, although patients are warned that their risk of relapse will increase modestly after they discontinue drugs. My recommendation is based on concerns regarding long-term side effects of such drugs including lymphoma, nodular regenerative hyperplasia of the liver and chronic liver damage. If a patient does relapse, I advise that recommencement of the same immunomodulator drug is generally effective. Anti-tumour necrosis factor (TNF) therapy in IBD, commenced after failing all other therapies, generally needs to continue long-term for as long as the patient is still responsive. However, patients who have commenced anti-TNF therapy before institution of immunomodulator drugs may be bridged to the immunomodulator after remission is attained, generally after three to four months. Reintroduction of the same anti-TNF therapy, if the patient relapses, may be associated with loss of response due to the development of antibodies.

PA: What can a gastroenterologist do to improve patient compliance?

SG: It is really important for the physician to make a genuine effort to improve compliance because patients would take a physician's advice seriously. An IBD nurse specialist may reinforce the message. Table 1 presents some practical suggestions.

\section{REFERENCES}

1. Ghosh S, Mitchell R. Impact of inflammatory bowel disease on quality of life: Results of the European Federation of Crohn's and Ulcerative colitis Associations (EFFCA) patient survey. J Crohn's Colitis 2007;1:10-20.

2. Hawthorne AB, Rubin G, Ghosh S. Medication non-adherence in ulcerative colitis - a review of strategies to improve adherence with mesalazine and other maintenance therapies. Aliment Pharmacol Ther 2008;27:1157-66.

3. Arnott IDR, Watts D, Ghosh S. Is clinical remission the optimum therapeutic goal in the treatment of Crohn's disease? Aliment Pharmacol Ther 2002;16:857-60.

4. Ghosh S. The challenges of medication non-adherence in ulcerative colitis: Practical suggestions to help patients. J Crohn's Colitis 2008;2:97-8. 


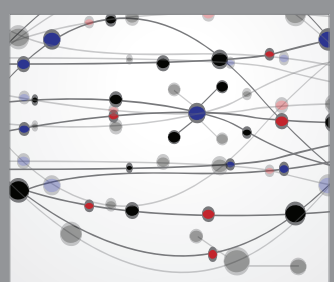

The Scientific World Journal
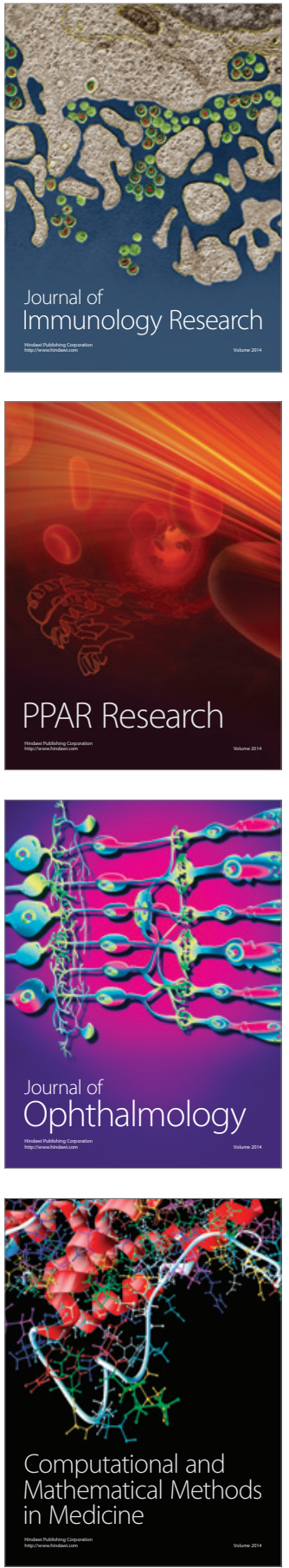

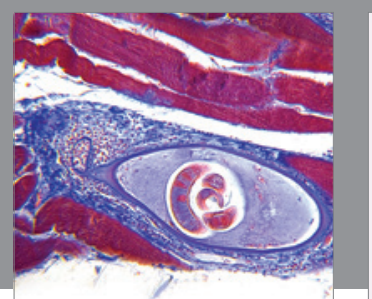

Gastroenterology Research and Practice

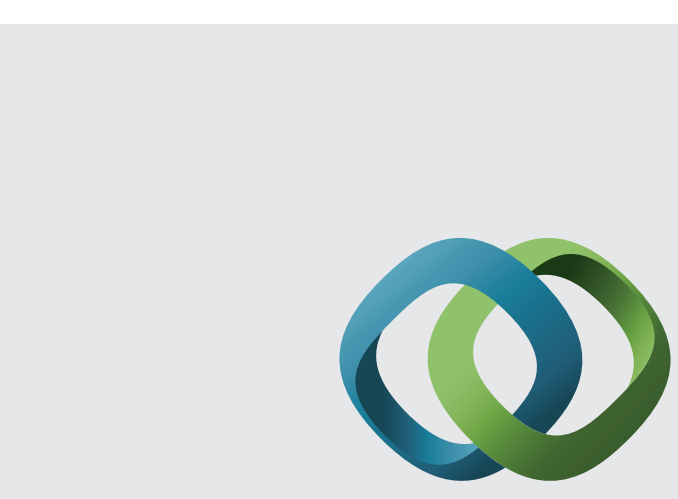

\section{Hindawi}

Submit your manuscripts at

http://www.hindawi.com
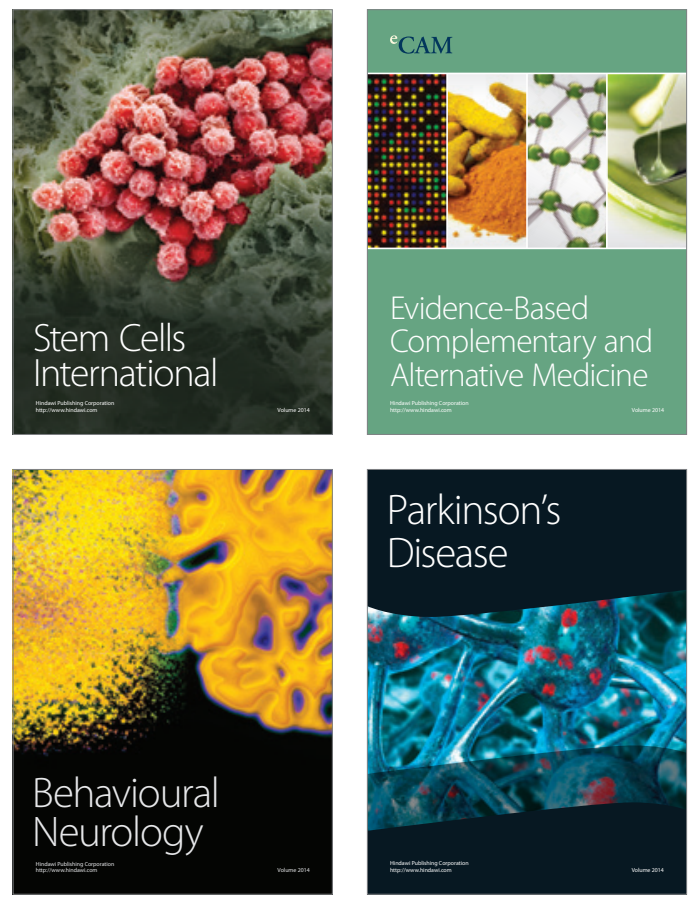
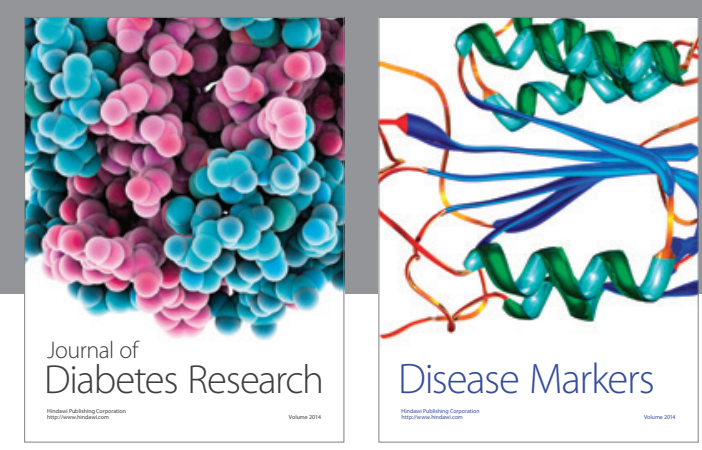

Disease Markers
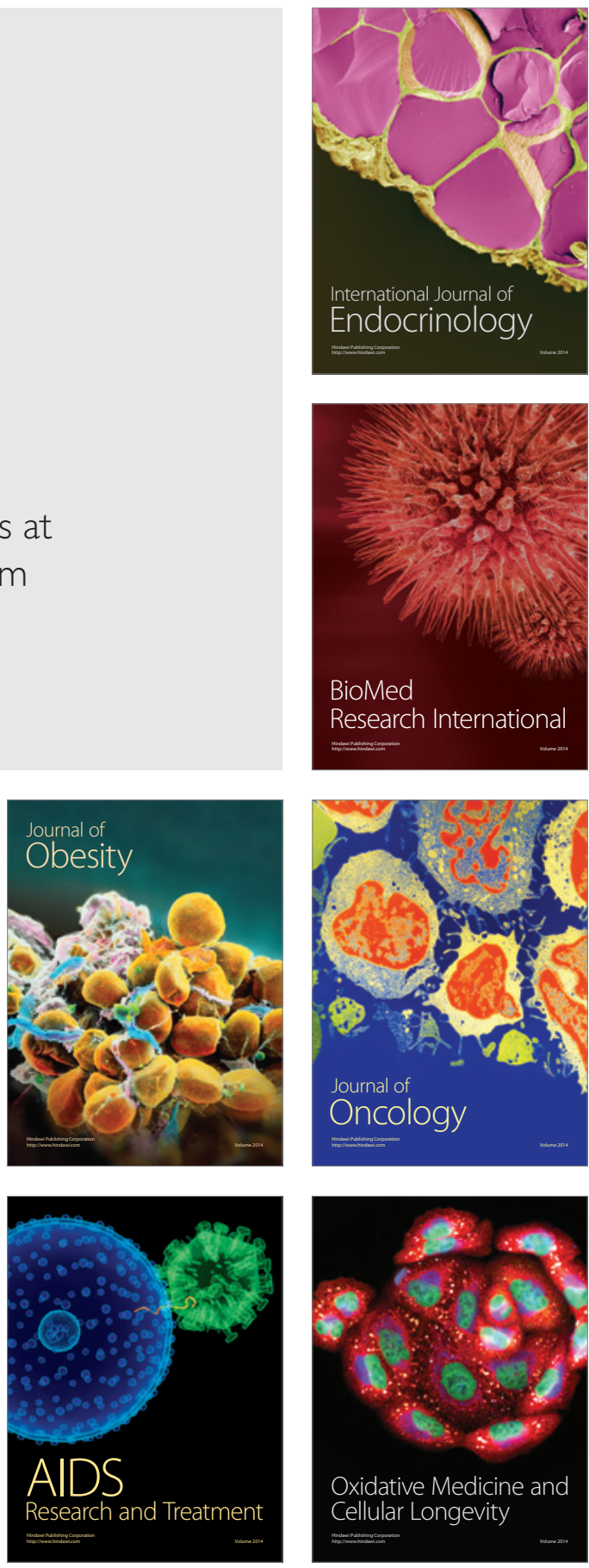